\title{
Designing and Evaluating Sociability in Online Video Games
}

\author{
Georgios Christou \\ European University Cyprus \\ 6 Diogenes St. \\ 1516, Nicosia, Cyprus \\ christoug@acm.org

\section{Effie Lai-Chong Law} \\ Department of Computer \\ Science \\ University of Leicester \\ LE1 7RH Leicester, U.K. \\ elaw@mcs.le.ac.uk

\section{David Geerts} \\ CUO | Social Spaces \\ KU Leuven / IBBT \\ Leuven, Belgium \\ david.geerts@soc.kuleuven.be
}

\section{Lennart Nacke}

University of Ontario Institute of

Technology

Ontario, Canada, L1H 7K4

lennart.nacke@uoit.ca

\section{Panayiotis Zaphiris}

Department of Multimedia and Graphic Arts

Cyprus University of Technology

Limassol, 84848, Cyprus

panayiotis.zaphiris@cut.ac.cy 


\section{Keywords}

Massively Multiplayer Online Games; Online Community; Sociability; Social Networks; User experience; Game experience; Player experience

\section{ACM Classification Keywords}

H5.3. Collaborative Computing: Online Games.

\section{Motivation}

This workshop builds on a successful SIG that was held during $\mathrm{CHI} 2011$ and that attracted about 30 people [2]. During this SIG, the point was raised that video game designers and developers do not have any tools that allow them to design and evaluate the sociability structures they place into their online games. This was identified as a major problem that requires a solution, as online video games are today more popular than email [4]. Since one of the major draws to these games is that they offer a new kind of "third-place" [5], a place where people can go and interact socially, which cannot be classified as home or work, tools that provide design guidelines for social structures and evaluate their power and efficacy are needed, so that designers can create structures that actually make a difference in the social dealings of their games' players.

The reality of designing social games is that our design knowledge is based on the functioning of online communities. Even though there have been several studies that examine the social structures in online video games, there have not yet been attempts to codify this knowledge, distill guidelines and principles, and create methods and frameworks that will allow professionals to create social games that are enhanced through the social experience rather than hindered. Seeing that trying to design for sociability may backfire
$[1,3]$ we need to examine the ways in which online game design can affect sociability. And to examine how social structures embedded in social games affect the player experience we need to create metrics and methods for evaluating sociability structures and the social player experience. Otherwise, the social interaction becomes instrumental [4], which may drive players away from an online game.

This workshop will focus on bringing together researchers and practitioners from various disciplines such as sociology, anthropology, computer science, human-computer interaction, psychology, computermediated communication, and others, to discuss the methods for design and evaluation of sociability in online video games. Questions to be addressed include, but are not limited to, the following [7]

- How do games augment or change existing social relationships?

- How do we design specifically for sociability?

- How do we evaluate sociability structures in online games?

- How do we leverage the sociability structures to create a "better" player experience?

This workshop also aims to further the understanding of the challenges relating to measures of sociability and player experience that are specific to online video games and to identify effective practical responses to these challenges.

\section{Workshop Goals}

The overarching goal of this workshop is to bring together academics and professionals that design, study, and evaluate social games, to create a framework that will include principles and guidelines for 
the design of social structures in online games, and metrics and methods for the evaluation of sociability structures and their impact on the player experience in online games. To achieve this, we propose the following sub-goals: first, to share experiences and practices, to create common understanding of particular methods of design and evaluation of social structures in online games. Once the methods and techniques are pooled together, recurrent themes will be identified and the beginnings of a framework that addresses these themes will be set. This leads to the second sub-goal: to set the basis for reaching a consensus on "best practices" for the design of social structures for online games. Third, evaluation methods and metrics will be pooled and their efficacy on the aforementioned social structures will be examined, to create a corpus of principles, methods, and metrics of the impact of the social structures on the player experience. Last, the resultant knowledge will be codified into a framework. Such a resultant framework will not only provide an industry standard, but will also allow the comparison of different designs to the social impact in different online video games.

For these reasons, we invite submissions that will include, but are not limited to, the following themes:

- Case studies of online game designs that have (not) succeeded in promoting sociability, with emphasis on their social structures.

- Studies of designing social structures in online games.

- Empirical studies on social structures in online games and their impact on the player experience.
- Experimental studies of metrics, methods, and techniques that have been used to evaluate social structures in online games.

- Conceptual frameworks that enable the evaluation of sociability in online video games.

- Studies providing connections between player experience and sociability in online video games.

The workshop will start with key presentations. The participants will then be divided into small groups, and guided by the key presentations, will be engaged in the following two activities: the first activity will be the examination of the case studies and experimental studies as above, and will try to distill certain design principles for social structures. The second activity will involve the analysis of conceptual frameworks, methods and metrics, and studies that enable the evaluation of the impact of social structures in games. During both activities the participants will also take into account how social structures impact player experience. The groups will then integrate their work, proposing design rules, principles, guidelines, methods and metrics as the basis of a framework that will provide an applied industrial standard towards the design and evaluation of social structures in online video games.

The proposed framework will help online game designers to create designs that leverage social structures to enhance - and evaluate those structures as to their impact on - player experience. It will also help the GUR (Game User Research) community by providing a guide that describes how certain design features may affect the player experience of online games. 


\section{Participants and Expected Community Interest}

As this is a workshop based on a previous successful SIG [2] that attracted about 30 participants, we expect that the workshop will appeal to both researchers and professionals from the wider GUR community. A key goal of this workshop is to enhance and promote the collaboration between, and increase the awareness of, researchers and practitioners that work in the field of online game design. A number of workshops and journal special issues have been held in recent years, such as "Computer Games / Players / Game Cultures: State and Perspectives of Digital Game Studies" [5], "Methods for evaluating games: how to measure usability and user experience in games?" [6], "HCI issues in games" [7], "Game User Research" [8], and others. These workshops and special issues have discussed issues of game design and player experience but not from the perspective of computer mediated communication among game players and its impact on the player experience. We believe that it is now a good time to discuss and integrate the approaches taken to design the social interaction that players experience when playing online games, especially as more people are using these games as a social meeting place, and not only as a game. Doing that will enable interested parties to collectively identify the specific approaches, tools, techniques and key problems that this new genre of games poses, as well as define guidelines for future solutions to these problems.

\section{References}

[1] Eklund, L. and Johansson, M. Social play? A study of social interaction in temporary group formation (PUG) in
World of Warcraft. Digital Games Research Association, City, 2010.

[2] Christou, G., Zaphiris, P., Ang, C. S. and Law, E. L.-C. Designing for the user experience of sociability in massively multiplayer online games. In Proceedings of the CHI 2011 annual conference extended abstracts on Human factors in computing systems (Vancouver, BC, Canada, 2011). ACM, [insert City of Publication],[insert 2011 of Publication].

[3] Ducheneaut, N., Moore, R. J. and Nickell, E. Designing for sociability in massively multiplayer games: an examination of the "third places" in SWG. IT University of Copenhagen, City, 2004

[4] Ducheneaut, N., Yee, N., Nickell, E. and Moore, R. J. "Alone together?": exploring the social dynamics of massively multiplayer online games. ACM Press, City, 2006.

[5] Computer Games / Players / Game Cultures: State and Perspectives of Digital Game Studies. City, 2009.

[6] Bernhaupt, R., Eckschlager, M. and Tscheligi, M. Methods for evaluating games: how to measure usability and user experience in games? ACM Press, City, 2007.

[7] Zaphiris, P. and Ang, C. S. Special Issue: HCI in Computer Games. Interacting With Computers, 19, 2 2007), 135-304

[8] El-Nasr, M. S., Desurvire, H., Nacke, L., Drachen, A., Calvi, L., Isbister, K. and Bernhaupt, R. Game user research. In Proceedings of the Proceedings of the 2012 ACM annual conference extended abstracts on Human Factors in Computing Systems Extended Abstracts (Austin, Texas, USA, 2012). ACM, [insert City of Publication],[insert 2012 of Publication]. 